\title{
Second Order Duality in Multiobjective Programming With Generalized Convexity
}

\author{
Xiaoyan Gao \\ College of Science, Xi'an University of Science and Technology, Xi'an 710054, China \\ angang21@126.com
}

\begin{abstract}
The purpose of this paper is concerned with a class of multi objective programming problems in which the objective functions and constrained functions are twice differentiable and containing the support functions of a compact convex set. For such mathematic programming, to deal with its dual problem, first, the concept of second order $B-(p, r)-$ invexity is introduced. Further, the Mangasarian type second order dual model associated with the multi objective problem are formulated. Several weak, strong and strict converse dual theorems are established and proved by utilizing the new generalized convexity. The results extend and improve the corresponding results in the literature.
\end{abstract}

Keywords: Second order B - ( $p, r)$ - invexity, Multiobjective Programming, Mangasarian type second order dual, efficient solution

\section{Introduction}

The field of multi objective programming known as vector programming has grown remarkably in different directions in the setting of optimality conditions and duality theory since the 1980's under the assumptions of various generalized convex functions. See, for example, [1-3] and the references cited therein. In recent past, these results have been generalized by many authors. Jayswal, et al., [4] established the sufficient optimality conditions and duality results under the assumptions of $(p, r)-\rho-(\eta, \theta)-$ invexity. In the papers [5-8], the multi objective programming problems were discussed under the generalized convexity assumptions. Slimani, et al., [9] formulated non differentiable multi objective programming involving generalized $d$-invexity.

Duality plays a fundamental role in mathematics, especially in optimization; see for example Ref. [10-12]. It is an old study, additionally to a given optimization problem, a corresponding dual problem. Duality has resulted in many applications within optimization, and it has provided many unifying conceptual insights into economics and management science. Duality received more attention and many researchers have contributed to the development of duality in optimization. During the past decades, the common duality was extended to some classes of second-order duality under the assumptions of higher-order convexities. More specifically, in [13], the concept of second order generalized $\alpha$ - type I univex function were introduced and several duality theorems were obtained. Gupta, et al., [14] formulated the symmetric duality for multi objective programming problems by utilizing higher-order $(F, \alpha, \rho, d)$ - convexity. Also, we can see the references in Ref. [15].

In this paper, motivated by the above work, we first introduce new generalized invex functions, called second order $B-(p, r)$ - invex functions. Further, we develop the dual theory. Mangasarian type second order dual for a class of multi objective programming 
problems involving support functions are considered. Several dual results for the dual problems in which the functions occurring belong to the class of second order $B-(p, r)-$ invex functions

\section{Notations and Preliminaries}

The following convention of vectors $R^{n}$ will be followed throughout this paper: $x \geqq y \Leftrightarrow x_{i} \geqq y_{i}, i=1,2, \cdots, n ; x \geq y \Leftrightarrow x \geqq y, x \neq y ; x>y \Leftrightarrow x_{i}>y_{i}, i=1,2, \cdots, n$.

Let $p, r$ be arbitrary real numbers. $\eta, \theta: X \times X \rightarrow R^{n}, b: X \times X \rightarrow R_{+}$, $\alpha: X \times X \rightarrow R_{+} \backslash\{0\}$, and the scalar function $\varphi: X \rightarrow R$ be twice differentiable at $\bar{x} \in X$ and $\rho \in R$.

Definition2.1. The function $\varphi$ is said to be second order $B-(p, r)-$ invex $\bar{x}$ on $X$, if for all $x \in X$, there exist $b, \alpha, \eta, \theta, \rho$ and $q \in R^{n}$, such that

$$
\begin{aligned}
& \frac{1}{r} b(x, \bar{x})\left[e^{r\left(\varphi(x)-\varphi(\bar{x})+\frac{1}{2} q^{T} \nabla^{2} \varphi(\bar{x}) q\right)}-1\right] \\
& \geqq \frac{1}{p} \alpha(x, \bar{x})\left[\nabla \varphi(\bar{x})+\nabla^{2} \varphi(\bar{x}) q\right]^{T}\left(e^{p \eta(x, \bar{x})}-I\right)+\rho\|\theta(x, \bar{x})\|^{2} \\
& \text { for } p \neq 0, r \neq 0
\end{aligned}
$$

If for all $x \in X, x \neq \bar{x}$, the above inequality holds as strict inequality, then $\varphi$ is said to be strictly second order $B-(p, r)-$ invex at $\bar{x}$ on $X$.

Definition2.2. The function $\varphi$ is said to be second order $B-(p, r)-$ pseudoinvex $\bar{x}$ on $X$, if for all $x \in X$, there exist $b, \alpha, \eta, \theta, \rho$ and $q \in R^{n}$, such that

$$
\begin{aligned}
& \frac{1}{r} b(x, \bar{x})\left[e^{r\left(\varphi(x)-\varphi(\bar{x})+\frac{1}{2} q^{T} \nabla^{2} \varphi(\bar{x}) q\right)}-1\right]<0 \\
& \Rightarrow \frac{1}{p} \alpha(x, \bar{x})\left[\nabla \varphi(\bar{x})+\nabla^{2} \varphi(\bar{x}) q\right]^{T}\left(e^{p \eta(x, \bar{x})}-I\right)<-\rho\|\theta(x, \bar{x})\|^{2} \\
& \text { for } p \neq 0, r \neq 0
\end{aligned}
$$

Definition2.3. The function $\varphi$ is said to be strictly second order $B-(p, r)-$ pseudoinvex $\bar{x}$ on $X$, if for all $x \in X, x \neq \bar{x}$, there exist $b, \alpha, \eta, \theta, \rho$ and $q \in R^{n}$, such that

$$
\begin{aligned}
& \frac{1}{p} \alpha(x, \bar{x})\left[\nabla \varphi(\bar{x})+\nabla^{2} \varphi(\bar{x}) q\right]^{T}\left(e^{p \eta(x, \bar{x})}-I\right) \geqq-\rho\|\theta(x, \bar{x})\|^{2} \\
& \Rightarrow \frac{1}{r} b(x, \bar{x})\left[e^{r\left(\varphi(x)-\varphi(\bar{x})+\frac{1}{2} q^{T} \nabla^{2} \varphi(\bar{x}) q\right)}-1\right]>0 \\
& \text { for } p \neq 0, r \neq 0
\end{aligned}
$$

Or equivalently 


$$
\begin{aligned}
& \frac{1}{r} b(x, \bar{x})\left[e^{r\left(\varphi(x)-\varphi(\bar{x})+\frac{1}{2} q^{T} \nabla^{2} \varphi(\bar{x}) q\right)}-1\right] \leqq 0 \\
& \Rightarrow \frac{1}{p} \alpha(x, \bar{x})\left[\nabla \varphi(\bar{x})+\nabla^{2} \varphi(\bar{x}) q\right]^{T}\left(e^{p \eta(x, \bar{x})}-I\right)<-\rho\|\theta(x, \bar{x})\|^{2} \\
& \text { for } p \neq 0, r \neq 0
\end{aligned}
$$

Definition2.4. The function $\varphi$ is said to be second order $B-(p, r)-$ quasiinvex $\bar{x}$ on $X$, if for all $x \in X$, there exist $b, \alpha, \eta, \theta, \rho$ and $q \in R^{n}$, such that

$$
\begin{aligned}
& \frac{1}{r} b(x, \bar{x})\left[e^{r\left(\varphi(x)-\varphi(\bar{x})+\frac{1}{2} q^{T} \nabla^{2} \varphi(\bar{x}) q\right)}-1\right] \leqq 0 \\
& \Rightarrow \frac{1}{p} \alpha(x, \bar{x})\left[\nabla \varphi(\bar{x})+\nabla^{2} \varphi(\bar{x}) q\right]^{T}\left(e^{p \eta(x, \bar{x})}-I\right) \leqq-\rho\|\theta(x, \bar{x})\|^{2} \\
& \text { for } p \neq 0, r \neq 0
\end{aligned}
$$

Or equivalently

$$
\begin{aligned}
& \frac{1}{p} \alpha(x, \bar{x})\left[\nabla \varphi(\bar{x})+\nabla^{2} \varphi(\bar{x}) q\right]^{T}\left(e^{p \eta(x, \bar{x})}-I\right)>-\rho\|\theta(x, \bar{x})\|^{2} \\
& \Rightarrow \frac{1}{r} b(x, \bar{x})\left[e^{r\left(\varphi(x)-\varphi(\bar{x})+\frac{1}{2} q^{T} \nabla^{2} \varphi(\bar{x}) q\right)}-1\right]>0 \\
& \text { for } p \neq 0, r \neq 0
\end{aligned}
$$

Remark2.1. It should be pointed out that the exponentials appearing on the right-hand sides of inequalities above are understood to be taken component wise, for example $e^{\left(a_{1}, a_{2}, \cdots, a_{n}\right)}=\left(e^{a_{1}}, e^{a_{2}}, \cdots e^{a_{n}}\right)$ and $I=(1,1, \cdots, 1) \in R^{n}$.

Remark2.2. All theorems in the further part of this work will be proved only in the case when $p \neq 0, r \neq 0$ (other cases are dealt with likewise since the only changes arise from the form of inequality defining the class of second-order $B-(p, r)-V$-type I for given $p$ and $r$ ). The proofs in the other cases are easier than in this one. Moreover, without limiting generality of considerations, we shall assume that $r>0$ (in the case when $r<0$, the directions some of the inequalities in the proofs of theorems should be changed to the opposite one).

It is well known that if a point $x_{0} \in X_{0}$ is an efficient solution for (MP) and the Slater's constraint qualification is satisfied on $X$, then the following Karush-Kuhn-Tucker type conditions are satisfied: there exist multipliers $\lambda \in R_{+}^{k}, \mu \in R_{+}^{m}, w_{i} \in R^{n}$ and $v_{j} \in R^{n}$ for $i \in K$ and $j \in M$, such that 


$$
\begin{aligned}
& \sum_{i=1}^{k} \lambda_{i} \nabla\left(f_{i}\left(x_{0}\right)+\left\langle w_{i}, x_{0}\right\rangle\right)+\sum_{j=1}^{m} \mu_{j} \nabla\left(g_{j}\left(x_{0}\right)+\left\langle v_{j}, x_{0}\right\rangle\right)=0 \\
& \sum_{j=1}^{m} \mu_{j}\left(g_{j}\left(x_{0}\right)+\left\langle v_{j}, x_{0}\right\rangle\right)=0 \\
& \left\langle w_{i}, x_{0}\right\rangle=s\left(x_{0} \mid C_{i}\right), w_{i} \in C_{i}, i \in K \\
& \left\langle v_{j}, x_{0}\right\rangle=s\left(x_{0} \mid D_{j}\right), v_{j} \in D_{j}, j \in M \\
& \lambda_{i}>0, \sum_{i=1}^{k} \lambda_{i}=1, \mu_{j} \geqq 0, j \in M
\end{aligned}
$$

Throughout this paper, we consider the following multi objective programming problem:

$$
\begin{aligned}
& \operatorname{minimize} F(x)=\left(f_{1}(x)+s\left(x \mid C_{1}\right), f_{2}(x)+s\left(x \mid C_{2}\right), \cdots, f_{k}(x)+s\left(x \mid C_{k}\right)\right) \\
& \text { s.t. } x \in X_{0}=\left\{x \in X: g_{j}(x)+s\left(x \mid D_{j}\right) \leqq 0, j \in M=\{1,2, \cdots, m\}\right\}
\end{aligned}
$$

Where $X \subseteq R^{n}$ is an open set. $f_{i}: X \rightarrow R, i=1,2, \cdots k$ and $g_{j}: X \rightarrow R, j \in M$ are twice differentiable on $X$ and $C_{i}, D_{j}$ are compact convex sets of $R^{n}$ for each $i \in K$ and $j \in M$, $s\left(x \mid C_{i}\right)$ and $s\left(x \mid D_{j}\right)$ denote the support functions of $C_{i}$ and $D_{j}$ evaluated at $X$, defined by

$$
s\left(x \mid C_{i}\right)=\max \left\{\left\langle w_{i}, x\right\rangle \mid w_{i} \in C_{i}\right\}, i \in K, s\left(x \mid D_{j}\right)=\max \left\{\left\langle v_{j}, x\right\rangle \mid v_{j} \in D_{j}\right\}, j \in M .
$$

Definition2.5. A point $\bar{x} \in X_{0}$ is an efficient solution for problem (MP), if and only if there exists no $x \in X_{0}$, such that

$$
f_{i}(x)+s\left(x \mid C_{i}\right) \leqq f_{i}(\bar{x})+s\left(\bar{x} \mid C_{i}\right) \text {, for all } i \in K
$$

And

$$
f_{l}(x)+s\left(x \mid C_{l}\right)<f_{l}(\bar{x})+s\left(\bar{x} \mid C_{l}\right) \text {, for at least one } i \in K
$$

Definition2.6. Let $\bar{x} \in X_{0}$ be an efficient solution for problem (MP), if there exists $(\bar{\lambda}, \bar{\mu}, \bar{w}, \bar{v})$, such that

$$
\begin{aligned}
& \sum_{i=1}^{k} \bar{\lambda}_{i}\left(\nabla f_{i}(\bar{x})+\bar{w}_{i}\right)+\sum_{j=1}^{m} \bar{\mu}_{j}\left(\nabla g_{j}(\bar{x})+\bar{v}_{j}\right)=0 \\
& \sum_{j=1}^{m} \bar{\mu}_{j} g_{j}(\bar{x})=0, \bar{\lambda}_{i}>0, \sum_{i=1}^{k} \bar{\lambda}_{i}=1, \bar{\mu}_{j} \geqq 0, j \in M
\end{aligned}
$$

Then $(\bar{x}, \bar{\lambda}, \bar{\mu}, \bar{w}, \bar{v})$ is said to be a Karush-Kuhn-Tucker point of (MP).

\section{Mangasarian Type Second Duality}

In this section, we consider the following Mangasarian type second order dual associated with multi objective problem (MP) and establish several duality theorems under the new function assumptions: 


$$
\begin{aligned}
& \max \text { imize }\left\{f_{1}(y)+\left\langle w_{1}, y\right\rangle-\frac{1}{2} q^{T} \nabla^{2} f_{1}(y) q+\sum_{j=1}^{m} \mu_{j}\left(g_{j}(y)+\left\langle v_{j}, y\right\rangle-\frac{1}{2} q^{T} \nabla^{2} g_{j}(y) q\right),\right. \\
& \left.\cdots, f_{k}(y)+\left\langle w_{k}, y\right\rangle-\frac{1}{2} q^{T} \nabla^{2} f_{k}(y) q+\sum_{j=1}^{m} \mu_{j}\left(g_{j}(y)+\left\langle v_{j}, y\right\rangle-\frac{1}{2} q^{T} \nabla^{2} g_{j}(y) q\right)\right\} \\
& \text { (MD) s.t. } \lambda_{i}>0, \sum_{i=1}^{k} \lambda_{i}=1, \mu_{j} \geqq 0, \\
& w_{i} \in C_{i}, v_{j} \in D_{j}, i \in K, j \in M \\
& y \in X, q \in R^{n}
\end{aligned}
$$

Theorem 3.1. (Weak Duality) Let $x$ and $(y, \lambda, \mu, w, v, q)$ be feasible solutions of (MP) and (MD), respectively such that

(i) $\left(f_{i}(\cdot)+\left\langle w_{i}, \cdot\right\rangle+\sum_{j=1}^{m} \mu_{j}\left(g_{j}(\cdot)+\left\langle v_{j}, \cdot\right\rangle\right)\right)$ is second-order $B-(p, r)-$ invex at $y$ with respect to $b_{i}, \alpha_{i}$ and $\rho_{i}$;

(ii) $b_{i}(x, y)>0$ for all $x \in D$;

(iii) $\sum_{i=1}^{k} \frac{\lambda_{i} \rho_{i}}{\alpha_{i}(x, y)} \geqq 0$ for all $x \in D$;

Then the following can not hold :

$$
f_{i}(x)+s\left(x \mid C_{i}\right) \leqq f_{i}(y)+\left\langle w_{i}, y\right\rangle-\frac{1}{2} q^{T} \nabla^{2} f_{i}(y) q+\sum_{j=1}^{m} \mu_{j}\left(g_{j}(y)+\left\langle v_{j}, y\right\rangle-\frac{1}{2} q^{T} \nabla^{2} g_{j}(y) q\right) \quad,
$$

for all $i \in K$

And

$$
f_{l}(x)+s\left(x \mid C_{l}\right)<f_{l}(y)+\left\langle w_{l}, y\right\rangle-\frac{1}{2} q^{T} \nabla^{2} f_{l}(y) q+\sum_{j=1}^{m} \mu_{j}\left(g_{j}(y)+\left\langle v_{j}, y\right\rangle-\frac{1}{2} q^{T} \nabla^{2} g_{j}(y) q\right) \quad,
$$

for at least one $l \in K$.

Proof: Suppose contrary to the result that the inequalities (10) and (11) hold.

In view of $\left\langle w_{i}, x\right\rangle \leqq s\left(x \mid C_{i}\right)$ with $i \in K, w_{i} \in C_{i}$, the inequalities (10) and (11) yield

$$
f_{i}(x)+\left\langle w_{i}, x\right\rangle \leqq f_{i}(y)+\left\langle w_{i}, y\right\rangle-\frac{1}{2} q^{T} \nabla^{2} f_{i}(y) q+\sum_{j=1}^{m} \mu_{j}\left(g_{j}(y)+\left\langle v_{j}, y\right\rangle-\frac{1}{2} q^{T} \nabla^{2} g_{j}(y) q\right) \quad,
$$

for all $i \in K$

And

$$
f_{l}(x)+\left\langle w_{l}, x\right\rangle\left\langle f_{l}(y)+\left\langle w_{l}, y\right\rangle-\frac{1}{2} q^{T} \nabla^{2} f_{l}(y) q+\sum_{j=1}^{m} \mu_{j}\left(g_{j}(y)+\left\langle v_{j}, y\right\rangle-\frac{1}{2} q^{T} \nabla^{2} g_{j}(y) q\right),\right. \text { for }
$$

at least one $l \in K$.

The hypothesis (i) implies 


$$
\begin{aligned}
& \frac{1}{r} b_{i}(x, y)\left[e^{\left.r\left(f_{i}(x)+\left\langle w_{i}, x\right\rangle+\sum_{j=1}^{m} \mu_{j}\left(g_{j}(x)+\left\langle v_{j}, x\right\rangle\right)-f_{i}(y)-\left\langle w_{i}, y\right\rangle-\sum_{j=1}^{m} \mu_{j}\left(\nabla g_{j}(y)+\left\langle v_{j}, y\right\rangle\right)+\frac{1}{2} q^{T} \nabla^{2} f_{i}(y) q\right)+\frac{1}{2} q^{T} \nabla^{2}\left(\sum_{j=1}^{m} \mu_{j} g_{j}(y)\right) q\right)}-1\right] \\
& \geqq \frac{1}{p} \alpha_{i}(x, y)\left[\nabla f_{i}(y)+w_{i}+\nabla^{2} f_{i}(y) q+\sum_{j=1}^{m} \mu_{j}\left(\nabla g_{j}(y)+v_{j}+\nabla^{2} g_{j}(y) q\right)\right]^{T}\left(e^{p \eta(x, y)}-I\right) \\
& +\rho_{i}\|\theta(x, y)\|^{2}, i \in K
\end{aligned}
$$

Since $b_{i}(x, y)>0, \mu_{j} \geqq 0,\left\langle v_{j}, x\right\rangle \leqq s\left(x \mid D_{j}\right), j \in M, v_{j} \in D_{j} \quad\left\langle w_{i}, x\right\rangle \leqq s\left(x \mid C_{i}\right)$, with the feasibility of $x$ for (MP), and the inequalities $(12,13)$, we have

$$
\begin{aligned}
& \frac{1}{-} b_{i}(x, y)\left[e^{r\left(f_{i}(x)+\left\langle w_{i}, x\right\rangle+\sum_{j=1}^{m} \mu_{j}\left(g_{j}(x)+\left\langle v_{j}, x\right\rangle\right)-f_{i}(y)-\left\langle w_{i}, y\right\rangle-\sum_{j=1}^{m} \mu_{j}\left(g_{j}(y)+\left\langle v_{j}, y\right\rangle\right)+\frac{1}{2} q^{T} \nabla^{2} f_{i}(y) q+\frac{1}{2} q^{T} \nabla^{2}\left(\sum_{j=1}^{m} \mu_{j} g_{j}(y) q\right)\right.}-1\right] \\
& \leqq 0
\end{aligned}
$$

And the above inequality holds as strictly inequality for at least one $i \in K$.

The inequality (14) along with (15) and $\lambda_{i}>0, \sum_{i=1}^{k} \lambda_{i}=1$ implies

$$
\begin{aligned}
& \frac{1}{p}\left[\sum_{i=1}^{k} \lambda_{i}\left(\nabla f_{i}(y)+w_{i}+\nabla^{2} f_{i}(y) q\right)+\sum_{j=1}^{m} \mu_{j}\left(\nabla g_{j}(y)+v_{j}+\nabla^{2} g_{j}(y) q\right)\right]^{T}\left(e^{p \eta(x, \bar{x})}-I\right) \\
& +\sum_{i=1}^{k} \frac{\lambda_{i} \rho_{i}}{\alpha_{i}(x, y)}\|\theta(x, y)\|^{2}<0, i \in K
\end{aligned}
$$

Which together with the first dual constraint condition yields

$$
\sum_{i=1}^{k} \frac{\lambda_{i} \rho_{i}}{\alpha_{i}(x, y)}\|\theta(x, y)\|^{2}<0
$$

That is

$$
\sum_{i=1}^{k} \frac{\lambda_{i} \rho_{i}}{\alpha_{i}(x, y)}<0
$$

A contradiction to the hypothesis (iii). Hence, the proof is completed.

Theorem 3.2. (Weak Duality) Let $x$ and $(y, \lambda, \mu, w, v, q)$ be feasible solutions of (MP) and (MD), respectively such that

(i) $\left[\sum_{i=1}^{k} \lambda_{i}\left(f_{i}(\cdot)+\left\langle w_{i}, \cdot\right\rangle\right)+\sum_{j=1}^{m} \mu_{j}\left(g_{j}(\cdot)+\left\langle v_{j}, \cdot\right\rangle\right)\right]$ is strictly second-order $B-(p, r)-$ invex at $y$ with respect to $b, \alpha$ and $\rho$;

(ii) $b(x, y) \geqq 0$ for all $x \in D$;

(iii) $\frac{\rho}{\alpha(x, y)} \geqq 0$ for all $x \in D$;

Then the following can not hold :

$$
f_{i}(x)+s\left(x \mid C_{i}\right) \leqq f_{i}(y)+\left\langle w_{i}, y\right\rangle-\frac{1}{2} q^{T} \nabla^{2} f_{i}(y) q+\sum_{j=1}^{m} \mu_{j}\left(g_{j}(y)+\left\langle v_{j}, y\right\rangle-\frac{1}{2} q^{T} \nabla^{2} g_{j}(y) q\right),
$$

for all $i \in K$

And 


$$
f_{l}(x)+s\left(x \mid C_{l}\right)<f_{l}(y)+\left\langle w_{l}, y\right\rangle-\frac{1}{2} q^{T} \nabla^{2} f_{l}(y) q+\sum_{j=1}^{m} \mu_{j}\left(g_{j}(y)+\left\langle v_{j}, y\right\rangle-\frac{1}{2} q^{T} \nabla^{2} g_{j}(y) q\right),
$$

for at least one $l \in K$.

Proof: We proceed by contradiction. Suppose that the inequalities (10) and (11) hold.

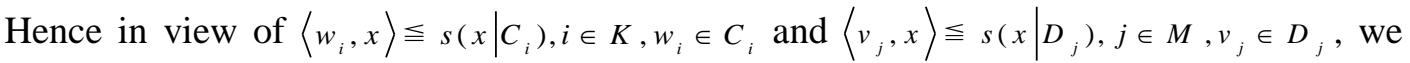
have

$$
f_{i}(x)+\left\langle w_{i}, x\right\rangle \leqq f_{i}(x)+s\left(x \mid C_{i}\right), i \in K \quad ; g_{j}(x)+\left\langle v_{j}, x\right\rangle \leqq g_{j}(x)+s\left(x \mid D_{j}\right), j \in M
$$

Since $\lambda_{i}>0, \sum_{i=1}^{k} \lambda_{i}=1, \mu_{j} \geqq 0, j \in M$, which along with the feasibility of $x$ for (MP), the inequalities (19) and (20) yield

$$
\begin{aligned}
& \sum_{i=1}^{k} \lambda_{i}\left(f_{i}(x)+\left\langle w_{i}, x\right\rangle\right)+\sum_{j=1}^{m} \mu_{j}\left(g_{j}(x)+\left\langle v_{j}, x\right\rangle\right)-\sum_{i=1}^{k} \lambda_{i}\left(f_{i}(y)+\left\langle w_{i}, y\right\rangle-\frac{1}{2} q^{T} \nabla^{2} f_{i}(y) q\right) \\
& -\sum_{j=1}^{m} \mu_{j}\left(g_{j}(y)+\left\langle v_{j}, y\right\rangle-\frac{1}{2} q^{T} \nabla^{2} g_{j}(y) q\right)<0
\end{aligned}
$$

Using the hypothesis (ii) and (i), the inequality (22) implies

$$
\begin{aligned}
& \frac{1}{p} \alpha(x, y)\left[\sum_{i=1}^{k} \lambda_{i}\left(\nabla f_{i}(y)+w_{i}+\nabla^{2} f_{i}(y) q\right)+\sum_{j=1}^{m} \mu_{j}\left(\nabla g_{j}(y)+v_{j}+\nabla^{2} g_{j}(y) q\right)\right]^{T}\left(e^{p \eta(x, y)}-I\right) \\
& +\rho\|\theta(x, y)\|^{2}<0
\end{aligned}
$$

Which is a contradiction with the feasibility of $(y, \lambda, \mu, w, v, q)$ for (MD). Hence we get the desired conclusion of theorem.

Theorem 3.3. (Weak Duality) Let $x$ and $(y, \lambda, \mu, w, v, q)$ be feasible solutions of (MP) and (MD), respectively such that

(i) $\left[\sum_{i=1}^{k} \lambda_{i}\left(f_{i}(\cdot)+\left\langle w_{i}, \cdot\right\rangle\right)+\sum_{j=1}^{m} \mu_{j}\left(g_{j}(\cdot)+\left\langle v_{j}, \cdot\right\rangle\right)\right]$ is second-order $B-(p, r)-$ pseudoinvex at $y$ with respect to $b, \alpha$ and $\rho$;

(ii) $b(x, y)>0$;

(iii) $\frac{\rho}{\alpha(x, y)} \geqq 0$ for all $x \in D$;

Then the following can not hold :

$$
f_{i}(x)+s\left(x \mid C_{i}\right) \leqq f_{i}(y)+\left\langle w_{i}, y\right\rangle-\frac{1}{2} q^{T} \nabla^{2} f_{i}(y) q+\sum_{j=1}^{m} \mu_{j}\left(g_{j}(y)+\left\langle v_{j}, y\right\rangle-\frac{1}{2} q^{T} \nabla^{2} g_{j}(y) q\right),
$$

for all $i \in K$

And

$$
f_{l}(x)+s\left(x \mid C_{l}\right)<f_{l}(y)+\left\langle w_{l}, y\right\rangle-\frac{1}{2} q^{T} \nabla^{2} f_{l}(y) q+\sum_{j=1}^{m} \mu_{j}\left(g_{j}(y)+\left\langle v_{j}, y\right\rangle-\frac{1}{2} q^{T} \nabla^{2} g_{j}(y) q\right) \quad,
$$

for at least one $l \in K$. 
Proof: Proceeding as in the proof of the above theorem, we get

$$
\frac{1}{r} b(x, y)\left[e^{\left.r\left(\sum_{i=1}^{k} \lambda_{i}\left(f_{i}(x)+\left\langle w_{i}, x\right\rangle\right)+\sum_{j=1}^{m} \mu_{j}\left(g_{j}(x)+\left\langle v_{j}, x\right\rangle\right)-\sum_{i=1}^{k} \lambda_{i}\left(f_{i}(y)+\left\langle w_{i}, y\right\rangle-\frac{1}{2} q^{T} \nabla^{2} f_{i}(y) q\right)-\sum_{j=1}^{m} \mu_{j}\left(g_{j}(y)+\left\langle v_{j}, y\right\rangle\right)-\frac{1}{2} q^{T} \nabla^{2} g_{j}(y) q\right)\right)}-1\right]<0
$$

In view of the hypothesis (i), the above inequality yields

$$
\begin{aligned}
& \frac{1}{p} \alpha(x, y)\left[\sum_{i=1}^{k} \lambda_{i}\left(\nabla f_{i}(y)+w_{i}+\nabla^{2} f_{i}(y) q\right)+\sum_{j=1}^{m} \mu_{j}\left(\nabla g_{j}(y)+v_{j}+\nabla^{2} g_{j}(y) q\right)\right]^{T}\left(e^{p \eta(x, y)}-I\right) \\
& +\rho\|\theta(x, y)\|^{2}<0
\end{aligned}
$$

Hence from the hypothesis (iii), we have

$$
\frac{1}{p}\left[\sum_{i=1}^{k} \lambda_{i}\left(\nabla f_{i}(y)+w_{i}+\nabla^{2} f_{i}(y) q\right)+\sum_{j=1}^{m} \mu_{j}\left(\nabla g_{j}(y)+v_{j}+\nabla^{2} g_{j}(y) q\right)\right]^{T}\left(e^{p \eta(x, y)}-I\right)<0
$$

Which is a contradiction with the feasibility of $(y, \lambda, \mu, w, v, q)$ for (MD). Hence we get the desired conclsion of theorem.

Theorem 3.4. (Weak Duality) Let $x$ and $(y, \lambda, \mu, w, v, q)$ be feasible solutions of (MP) and (MD), respectively such that

(i) $\left[f_{i}(\cdot)+\left\langle w_{i}, \cdot\right\rangle+\sum_{j=1}^{m} \mu_{j}\left(g_{j}(\cdot)+\left\langle v_{j}, \cdot\right\rangle\right)\right]$ is strictly second order $B-(p, r)-$ pseudoinvex at $y$ with respect to $b_{i}, \alpha_{i}$ and $\rho_{i}$;

(ii) $b_{i}(x, y) \geqq 0$, for all $x \in D$;

(iii) $\sum_{i=1}^{k} \frac{\lambda_{i} \rho_{i}}{\alpha_{i}(x, y)} \geqq 0$, for all $x \in D$;

Then the following can not hold :

$$
f_{i}(x)+s\left(x \mid C_{i}\right) \leqq f_{i}(y)+\left\langle w_{i}, y\right\rangle-\frac{1}{2} q^{T} \nabla^{2} f_{i}(y) q+\sum_{j=1}^{m} \mu_{j}\left(g_{j}(y)+\left\langle v_{j}, y\right\rangle-\frac{1}{2} q^{T} \nabla^{2} g_{j}(y) q\right) \quad,
$$

for all $i \in K$

And

$$
f_{l}(x)+s\left(x \mid C_{l}\right)<f_{l}(y)+\left\langle w_{l}, y\right\rangle-\frac{1}{2} q^{T} \nabla^{2} f_{l}(y) q+\sum_{j=1}^{m} \mu_{j}\left(g_{j}(y)+\left\langle v_{j}, y\right\rangle-\frac{1}{2} q^{T} \nabla^{2} g_{j}(y) q\right),
$$

for at least one $l \in K$.

Proof: Proceeding as in the proof of the theorem 3.1, by the hypothesis (i), the inequalities (12) and (13) together with the hypothesis (ii) imply

$$
\begin{aligned}
& \frac{1}{p} \alpha_{i}(x, y)\left[\nabla f_{i}(y)+w_{i}+\nabla^{2} f_{i}(y) q+\sum_{j=1}^{m} \mu_{j}\left(\nabla g_{j}(y)+v_{j}+\nabla^{2} g_{j}(y) q\right)\right]^{T}\left(e^{p \eta(x, y)}-I\right) \\
& +\rho_{i}\|\theta(x, y)\|^{2}<0
\end{aligned}
$$


Since $\lambda_{i}>0, \sum_{i=1}^{k} \lambda_{i}=1$, the above inequality impies

$$
\begin{aligned}
& \frac{1}{p}\left[\sum_{i=1}^{k} \lambda_{i}\left(\nabla f_{i}(y)+w_{i}+\nabla^{2} f_{i}(y) q\right)+\sum_{j=1}^{m} \mu_{j}\left(\nabla g_{j}(y)+v_{j}+\nabla^{2} g_{j}(y) q\right)\right]^{T}\left(e^{p \eta(x, y)}-I\right) \\
& +\sum_{i=1}^{k} \frac{\lambda_{i} \rho_{i}}{\alpha_{i}(x, y)}\|\theta(x, y)\|^{2}<0
\end{aligned}
$$

In view of the hypothesis (iii), the above inequality yields

$$
\frac{1}{p}\left[\sum_{i=1}^{k} \lambda_{i}\left(\nabla f_{i}(y)+w_{i}+\nabla^{2} f_{i}(y) q\right)+\sum_{j=1}^{m} \mu_{j}\left(\nabla g_{j}(y)+v_{j}+\nabla^{2} g_{j}(y) q\right)\right]^{T}\left(e^{p \eta(x, y)}-I\right)<0
$$

Which is a contradiction with the feasibility of $(y, \lambda, \mu, w, v, q)$ for (MD). Hence we get the desired conclusion of theorem.

Theorem 3.5. (Strong Duality) Assume that $\bar{x}$ is an efficient solution of (MP). Let $(\bar{x}, \bar{\lambda}, \bar{\mu}, \bar{w}, \bar{v})$ is a Karush-Kuhn-Tucker point of (MP). Then $(\bar{x}, \bar{\lambda}, \bar{\mu}, \bar{w}, \bar{v}, \bar{q}=0)$ is a feasible solution of (MD) and the two objectives have the same value. If, in addition, the assumptions of weak duality (theorem 3.1, theorem 3.2) hold for all the feasible solution of (MP) and (MD), then $(x, \bar{\lambda}, \bar{\mu}, \bar{w}, v, \bar{q}=0)$ is an efficient solution of (MD).

Proof: Since $(\bar{x}, \bar{\lambda}, \bar{\mu}, \bar{w}, \bar{v})$ is a Karush-Kuhn-Tucker point of (MP), then the first and second constraint conditions hold for $\bar{q}=0$. Therefore, $(\bar{x}, \bar{\lambda}, \bar{\mu}, \bar{w}, \bar{v}, \bar{q}=0)$ is a feasible solution of (MD) and the corresponding objectives values of (MP) and (MD) are equal. The efficiency of this feasible solution for (MD) thus follows weak duality (theorem 3.1, theorem 3.2).

Theorem 3.6. (Strict Converse Duality) Let $\bar{x}$ and $(\bar{u}, \bar{\lambda}, \bar{\mu}, \bar{w}, \bar{v}, \bar{q})$ be the feasible solutions of (MP) and (MD) respectively. Further, we suppose that

(i) $\sum_{i=1}^{k} \bar{\lambda}_{i}\left(f_{i}(\bar{x})+s\left(\bar{x} \mid C_{i}\right)\right)$

$$
\leqq \sum_{i=1}^{k} \bar{\lambda}_{i}\left(f_{i}(\bar{u})+\left\langle\bar{w}_{i}, \bar{u}\right\rangle-\frac{1}{2} \bar{q}^{T} \nabla^{2} f_{i}(\bar{u}) \bar{q}\right)+\sum_{j=1}^{m} \bar{\mu}_{j}\left(g_{j}(\bar{u})+\left\langle\bar{v}_{j}, \bar{u}\right\rangle-\frac{1}{2} \bar{q}^{T} \nabla^{2} g_{j}(\bar{u}) \bar{q}\right) ;
$$

(ii) $\left[\sum_{i=1}^{k} \bar{\lambda}_{i}\left(f_{i}(\cdot)+\left\langle\bar{w}_{i}, \cdot\right\rangle\right)+\sum_{j=1}^{m} \bar{\mu}_{j}\left(g_{j}(\cdot)+\left\langle\bar{v}_{j}, \cdot\right\rangle\right)\right]$ is strictly second order $B-(p, r)-$ invex at $\bar{u}$ with respect to $b, \alpha$ and $\rho$;

(iii) $b(x, \bar{u})>0$, for all $x \in D$;

(iv) $\rho \geqq 0$.

Then $\bar{x}=\bar{u}$, that is, $\bar{u}$ is an efficient solution of (MP).

Proof: We assume that $\bar{x} \neq \bar{u}$ and exhibit a contradiction.

In view of the hypothesis (ii), we have 


$$
\begin{aligned}
& \frac{1}{r} b(\bar{x}, \bar{u})\left[e^{r\left[\sum_{i=1}^{k} \bar{\lambda}_{i}\left(f_{i}(\bar{x})+\left\langle\bar{w}_{i}, \bar{x}\right\rangle\right)+\sum_{j=1}^{m} \bar{\mu}_{j}\left(g_{j}(\bar{x})+\langle\bar{v} j, \bar{x}\rangle\right)-\sum_{i=1}^{k} \bar{\lambda}_{i}\left(f_{i}(\bar{u})+\left\langle\bar{w}_{i}, \bar{u}\right\rangle-\frac{1}{2}-\bar{q} \nabla^{2} f_{i}(\bar{u}) \bar{q}\right)-\sum_{j=1}^{m} \bar{\mu}_{j}\left(g_{j}(\bar{u})+\left\langle\bar{v}_{j}, \bar{u}\right\rangle-\frac{1}{2} q^{-T} \nabla^{2} f_{i}(\bar{u}) \bar{q}\right)\right]}-1\right] \\
& >\frac{1}{p} \alpha(\bar{x}, \bar{u})\left[\sum_{i=1}^{k} \bar{\lambda}_{i}\left(\nabla f_{i}(\bar{u})+\bar{w}_{i}+\nabla^{2} f_{i}(\bar{u}) \bar{q}\right)+\sum_{j=1}^{m} \bar{\mu}_{j}\left(\nabla g_{j}(\bar{u})+\bar{v}_{j}+\nabla^{2} g_{j}(\bar{u}) \bar{q}\right)\right]^{T}\left(e^{p \eta(\bar{x}, \bar{u})}-I\right) \\
& +\rho\|\theta(\bar{x}, \bar{u})\|^{2}
\end{aligned}
$$

Which in view of the feasibility of $(\bar{u}, \bar{\lambda}, \bar{\mu}, \bar{w}, \bar{v}, \bar{q})$ for (MD) and the hypothesis (iv) implies

$$
\begin{aligned}
& \frac{1}{-b}(\bar{x}, \bar{u})\left[e^{r\left[\sum_{i=1}^{k} \bar{\lambda}_{i}\left(f_{i}(\bar{x})+\langle\bar{w} i, \bar{x}\rangle\right)+\sum_{j=1}^{m} \bar{\mu}_{j}\left(g_{j}(\bar{x})+\langle\bar{v}, \bar{x}\rangle\right)-\sum_{i=1}^{k} \bar{\lambda}_{i}\left(f_{i}(\bar{u})+\left\langle\overline{w_{i}}, \bar{u}\right\rangle-\frac{1}{2} q^{-T} \nabla^{2} f_{i}(\bar{u}) \bar{q}\right)-\sum_{j=1}^{m} \bar{\mu}_{j}\left(g_{j}(\bar{u})+\langle\bar{v} j, \bar{u}\rangle-\frac{1}{2}-\bar{q} \nabla^{2} f_{i}(\bar{u}) \bar{q}\right)\right]}-1\right] \\
& r r \\
& >0
\end{aligned}
$$

Which together with the hypothesis (iii), $\left\langle\bar{w}_{i}, \bar{x}\right\rangle \leqq s\left(\bar{x} \mid C_{i}\right)$ with $i \in K, \bar{w}_{i} \in C_{i}$ and the feasibility of $\bar{x}$ for (MP) yields

$$
\begin{aligned}
& \sum_{i=1}^{k} \bar{\lambda}_{i}\left(f_{i}(\bar{x})+s\left(\bar{x} \mid C_{i}\right)\right)-\sum_{i=1}^{k} \bar{\lambda}_{i}\left(f_{i}(\bar{u})+\left\langle\bar{w}_{i}, \bar{u}\right\rangle\right. \\
& \left.-\frac{1}{2} \bar{q}^{T} \nabla^{2} f_{i}(\bar{u}) \bar{q}\right)-\sum_{j=1}^{m} \bar{\mu}_{j}\left(g_{j}(\bar{u})+\left\langle\bar{v}_{j}, \bar{u}\right\rangle-\frac{1}{2} \bar{q}^{T} \nabla^{2} g_{j}(\bar{u}) \bar{q}\right)>0
\end{aligned}
$$

Which is a contradiction with the hypothesis (i). Hence, $\bar{x}=\bar{u}$. On the other hand, proceeding as in the proof of the theorem 3.2, we can obtain easily that $\bar{u}$ is an efficient solution of (MP).

\section{Discussion and Conclusion}

Throughout this paper, we have defined new classes of generalized convexity functions calling second order $B-(p, r)$ - invex functions which extending many wellknown classes of generalized convex functions. Furthermore, we have achieved and proved some dual results for Mangasarian type second order dual associated with multi objective problem under the new functions assumptions. There should be further opportunities for exploiting this structure of the multi objective programming problem.

\section{Acknowledgements}

This work is supported by Scientific Research Program Funded by Shaanxi Provincial Education Department (Program No. 12JK0875) and Scientific Research Development Program Funded by Xi' an University of Science and Technology ((Program No: 201349).

\section{References}

[1] M. A. Hanson, R. Pini and C. Singh, J. Math. Anal. Appl., vol. 2, no. 261, (2002).

[2] T. R. Gulati, I. Ahmad and D. Agarcual, J. Optim. Theory Appl., vol. 3, no. 135, (2007).

[3] S. K. Mishra, S. Y. Wang and K. K. Lai, J. Glob. Optim., vol. 4, no. 29, (2004).

[4] A. Jayswal, R. Kumar and D. Kumar, J. Appl. Math. Comput., (2012).

[5] G. An and X. Y. Gao, J. Comput. Theor. Nanosci., vol. 12, no. 10, (2013).

[6] X. Y. Long, J. Optim. Theory Appl., vol. 1, no. 148, (2011). 
[7] X. Y. Gao, J. theor. Appl. Inf. Technol., vol. 1, no. 46, (2012).

[8] C. Nahak and R. N. Mohapatra, Optim. Lett., vol. 2, no. 6, (2012).

[9] H. Slimani and M. S. Radjef, Eur. J. Oper. Res., vol. 1, no. 202, (2010).

[10] X. Y. Gao, Int. Rev. Comput. Softw., vol. 5, no. 7, (2012).

[11] D. S. Kim and H. J. Lee, J. Inequal. Appl., (2010).

[12] K. D. Bae, Y. M. Kang and D. S. Kim, J. Inequ. Appl., (2010).

[13] S, Sharma and T. R. Gulaati, J. Glob. Optim., vol. 1, no. 52, (2012).

[14] S. K. Gupta, N. Kailey and M. K. Sharma, J. Comput. Math. Appl., vol. 8, no. 60, (2010).

[15] I. Ahmad and Z. Husain, J. Appl. Anal., vol. 1, no. 14, (2010).

\section{Author}

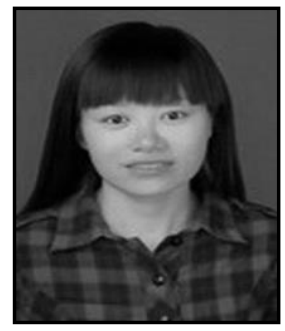

Xiaoyan Gao, she was born in shanxi Province, China on August 28, 1979. She received her master's degree in optimization theory and applications from Yan'an University, China in 2005. Her main research fields include the generalized convexity, the optimization theory and applications for semi-infinite and multi objective programming, etc. 
International Journal of Grid Distribution Computing Vol.7, No.5 (2014) 